\title{
ANOTHER THEOREM ON BOUNDED ANALYTIC FUNCTIONS
}

\author{
JEAN-PIERRE KAHANE
}

This note is an attempt to solve the conjecture stated at the end of the preceding paper [1]. We are able to prove the following.

THEOREM 1. Let $\left\{\phi_{n}\right\}$ be a sequence of summable functions on the circle such that

$$
l(f)=\lim _{n \rightarrow \infty} \int f \phi_{n}
$$

exists for all $f \in H^{\infty}$ (space of bounded functions on the circle with a positive spectrum; the integral is taken over the circle). Then there is a $\phi \in L^{1}$ such that

$$
l(f)=\int f \phi
$$

for all $f \in A$ (space of continuous functions on the circle with a positive spectrum).

Proof. As in [1] we see first that there exists a measure $d \mu$ on the circle such that $l(f)=\int f d \mu$ whenever $f \in A$. Let us prove that $d \mu$ is absolutely continuous.

Suppose that $d \mu$ is not absolutely continuous. Let $E$ be a closed set on the circle, with Lebesgue measure zero, such that $\int_{E} d \mu=\mu(E) \neq 0$. Let $h$ be a function in A such that $h=1$ on $E$ and $|h|<1$ outside (the existence of such a function is well known; it is used also in [1]). We have the following equalities $(m=1,2, \cdots ; n=1,2, \cdots)$ :

(1) $\lim _{m \rightarrow \infty} \int h^{m} d \mu=\mu(E)$,

(2) $\lim _{m \rightarrow \infty} \int h^{m} \phi_{n}=0$ for all $n$ 's,

(3) $\lim _{n \rightarrow \infty} \int h^{m} \phi_{n}=\int h^{m} d \mu$ for all $m$ 's.

If the sequence $m_{j}$ is rapidly increasing (meaning that $m_{j+1}$ is sufficiently large when $m_{j}$ is given), we have

$$
f=\sum_{j=1}^{\infty}(-1)^{j} h^{m_{i}} \in H^{\infty} .
$$

For, given $m_{j}$, we define $E_{j}$ as the set where $\left|h^{m_{j}}-1\right|<2^{-j}$, and we have $\left|h^{m_{j+1}}\right|<2^{-j}$ on $C E_{j}$ when $m_{j+1}$ is large enough. We shall write $L_{1}$ for this condition on the $m_{j}$.

Received by the editors July 10, 1966. 
We shall define by induction two sequences $m_{j}$ (satisfying $L_{1}$ ) and $n_{j}$. We shall use the formula

$$
\begin{aligned}
\int f \phi_{n_{j}} & =\sum_{k=1}^{j-1}(-1)^{k} \int h^{m_{k}} \phi_{n_{j}}+(-1)^{j} \int h^{m_{j}} \phi_{n_{j}}+\sum_{k=j+1}^{\infty}(-1)^{k} \int h^{m_{k}} \phi_{n_{j}} \\
& =A_{j}+B_{j}+C_{j} .
\end{aligned}
$$

We write $L_{2}$ for the condition

$$
\sum_{k=j+1}^{\infty}\left|\int h^{m_{k}} \phi_{n_{j}}\right|<\frac{1}{12}|\mu(E)| ;
$$

by (2), it is satisfied when $m_{j+1}, m_{j+2}, \cdots$ are chosen large enough, $n_{j}$ being given. We vrite $L_{3}$ for the condition

$$
\left|\int h^{m_{j}} d \mu\right|>\frac{11}{12}|\mu(E)| \text {; }
$$

by (1), it is satisfied when $m_{j}$ is large. Now suppose that $m_{1}, \cdots$, $m_{j-1}, n_{1}, \cdots, n_{j-1}$ are given in such a manner that the conditions $L_{1}, L_{2}, L_{3}$ are satisfied at this stage. They will be satisfied at the following stage if $m_{j}$ is sufficiently large, $m_{j} \geqq m_{j}^{*}$, say. We define $n_{j}^{*}$ so that $n \geqq n_{j}^{*}$ implies

$$
\left|A_{j}-A_{j}^{\infty}\right|<|\mu(E)| / 12
$$

where

$$
A_{j}^{\infty}=\sum_{k=1}^{j-1}(-1)^{k} \int h^{m_{k}} d \mu
$$

that is possible because of (3). Now we consider two cases, namely

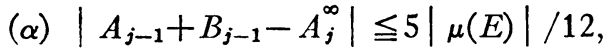

(B) $\left|A_{j-1}+B_{j-1}-A_{j}^{\infty}\right|>5|\mu(E)| / 12$.

In the case $(\alpha)$, we choose $m_{j}=m_{j}^{*}$, and $n_{j}$ large enough $\left(\geqq n_{j}^{*}\right)$ so that $\left|B_{j}\right|>11|\mu(E)| / 12$; that is possible because of (3) and $L_{3}$. In the case $(\beta)$, we choose $n_{j}=n_{j}^{*}$, and $m_{j}$ large enough $\left(\geqq m_{j}^{*}\right)$ so that $\left|B_{j}\right|$ $<|\mu(E)| / 12$; that is possible because of (2). In each case, we have

$$
\left|A_{j-1}+B_{j-1}-A_{j}-B_{j}\right|>3|\mu(E)| / 12 \text {. }
$$

Taking $L_{2}$ into account, we have $\left|C_{j-1}\right|$ and $\left|C_{j}\right|$ majorized by $|\mu(E)| / 12$, and therefore

$$
\left|\int f \phi_{n_{j-1}}-\int f \phi_{n_{j}}\right|>\frac{1}{12}|\mu(E)| .
$$


Therefore the sequence $\int f \phi_{n}$ is not convergent, against our assumption. The contradiction proves that $d \mu$ is absolutely continuous, that is $l(f)=\int f d \mu=\int f \phi$ whenever $f \in A$, for some $\phi \in L^{1}$.

REMARK. If $\phi_{n}(t)=\sum_{k=-\infty}^{\infty} a_{n, k} e^{-i k t}$, the assumption of the theorem is the existence of $\lim _{n \rightarrow \infty} \sum_{0}^{\infty} a_{n, k} b_{k}$ for all $\sum_{0}^{\infty} b_{k} e^{i k t} \in H^{\infty}$. The conclusion is $\lim _{n \rightarrow \infty} a_{n, k}=\int \phi(\theta) e^{i k \theta}$ for some $\phi \in L^{1}(k=0,1,2, \cdots)$. Theorem 1 of [1] follows as a particular case.

We are not able to prove that $l(f)=\int f \phi$ for all $f \in H^{\infty}$. Nevertheless, this holds for many functions in $H^{\infty}$. Precisely, we have

Theorem 2. Keeping the same notations as in Theorem 1 , let $D_{l}$ be the set of all $f \in H^{\infty}$ such that $l(f)=\int f \phi$, and let $D$ be the intersection of the $D_{l}$ for all $l$. Then $(\alpha) D_{l}$ is a closed subspace of $H^{\infty}$ and, given any $f \in H^{\infty}$, almost all translates of $f$ belong to $D_{l} .(\beta) D$ is a closed subalgebra of $H^{\infty}$, invariant under translation; it contains all $f \in H^{\infty}$ such that $f g \in D$ for some outer function $g \in D$; in particular, it contains all $f \in H^{\infty}$ which are continuous on the circle except on a closed set of measure zero.

Proof. We may suppose that the $\phi_{n}$ are trigonometric polynomials. By the Banach-Steinhaus theorem, the linear functionals $f \rightarrow \int f \phi_{n}$ are uniformly bounded on $A$. There exist measures $d \mu_{n}$, with bounded norms, such that $\int f \phi_{n}=\int f d \mu_{n}$ for all $f \in A$. By the F. and M. Riesz theorem (or another device) the $d \mu_{n}$ are absolutely continuous. Therefore we may suppose that the $\phi_{n}$ have bounded $L^{1}$-norms.

In order to prove $(\alpha)$ we may suppose $\phi=0$. The fact that $D_{l}$ is a closed subspace of $H^{\infty}$ is obvious. Given $f \in H^{\infty}$, we write $f_{s}(t)=f(t-s)$. Given $\psi \in L^{1}$, we have $f * \psi \in A$, and by Theorem 1

$$
\lim _{n \rightarrow \infty} \iint \phi_{n}(t) f(t-s) \psi(s) d s d t=0 .
$$

By assumption

$$
\lim _{n \rightarrow \infty} \int \phi_{n}(t) f(t-s) d t=l\left(f_{\bullet}\right)
$$

and since the $\phi_{n}$ have bounded $L^{1}$-norms, the integrals $\int \phi_{n}(t) f(t-s) d t$ are uniformly bounded with respect to $n$ and $s$. By the Lebesgue theorem

$$
\int l\left(f_{s}\right) \psi(s) d s=0
$$

and since $\psi$ is an arbitrary function in $L^{1}, l\left(f_{8}\right)=0$ for almost every $s$. That proves $(\alpha)$. 
In order to prove $(\beta)$ we write

$$
\begin{gathered}
\lim _{n \rightarrow \infty} \int f g \phi_{n}=l_{f}(g)=l_{g}(f)=l(f g) \quad\left(f \in H^{\infty}, g \in H^{\infty}\right), \\
l_{f}(g)=\int g \phi_{f} \quad \text { when } g \in D .
\end{gathered}
$$

We have $A \subset D$ as a reformulation of Theorem 1 .

Suppose $f \in A$. Taking $g \in A$, we have $f g \in A$. Since $f g \in D$ and $g \in D$, we have

$$
\begin{gathered}
\int f g \phi=l(f g)=l_{f}(g)=\int g \phi_{f}, \\
\int g\left(f \phi-\phi_{f}\right)=0
\end{gathered}
$$

and since $g$ is arbitrary in $A, f \phi=\phi_{f}\left(\bmod H_{0}^{\prime}\right)$ (meaning that the Fourier coefficients or order $\leqq 0$ are the same).

Now suppose $g \in D$. Taking $f \in A$ we have

$$
l(f g)=l_{f}(g)=\int g \phi_{f}=\int f g \phi
$$

since $f \phi=\phi_{f}\left(\bmod H_{0}^{\prime}\right)$. Therefore $f g \in D_{l}$ and, $l$ being arbitrary, $f g \in D$. Since $f g \in D$ and $g \in D$, we have

$$
\int f\left(g \phi-\phi_{0}\right)=0
$$

and since $f$ is arbitrary in $A, g \phi=\phi_{g}\left(\bmod H_{0}^{\prime}\right)$.

If $f \in D$ and $g \in D$, we still have (6) because $f \phi=\phi_{f}\left(\bmod H_{0}^{\prime}\right)$, and $f g \in D$ as a consequence. Therefore $D$ is a subalgebra of $H^{\infty}$. It is closed because each $D_{l}$ is closed, and it is obviously invariant under translation.

Finally, suppose that $f \in H^{\infty}, g \in D, g$ is an outer function and $f g \in D$. We still have (4) and (5). Moreover, since $D$ is an algebra, we have

$$
\int g h\left(f \phi-\phi_{f}\right)=0
$$

for all $h \in D$. Therefore $g\left(f \phi-\phi_{f}\right)=0\left(\bmod H_{0}^{\prime}\right)$. Since $g$ is an outer function, it follows that $f \phi=\phi_{f}\left(\bmod H_{0}^{\prime}\right)$. As a conclusion 


$$
l(f)=l_{f}(1)=\int \phi_{f}=\int f \phi,
$$

that is, $f \in D_{l}$, and since $l$ is arbitrary, $f \in D$.

Given a closed set $K$ of measure zero on the circle, there exists a continuous outer function $g$ vanishing on $K$ (that follows immediately from a proof of Fatou's theorem). If $f$ is continuous except on $K$, $f g \in A$, therefore $f \in D$. That ends the proof of Theorem 2 .

\section{REFERENCE}

1. George Piranian, A. L. Shields and J. H. Wells, Bounded analytic functions and absolutely continuous measures, Proc. Amer. Math. Soc. 18 (1967), pp., 818-826.

UNiversity of Chicago and

Faculte des Sciences d'Orsay 